Vietnam Journal of Mechanics, VAST, Vol. 42, No. 3 (2020), pp. $307-320$

DOI: https://doi.org/10.15625/0866-7136/15309

Dedicated to Professor J.N. Reddy on the Occasion of His $75^{\text {th }}$ Birthday

\title{
THERMAL POSTBUCKLING ANALYSIS OF FG-CNTRC DOUBLY CURVED PANELS WITH ELASTICALLY RESTRAINED EDGES USING REDDY'S HIGHER ORDER SHEAR DEFORMATION THEORY
}

\author{
Hoang Van Tung ${ }^{1, *}$, Nguyen Dinh Kien ${ }^{2}$, Le Thi Nhu Trang ${ }^{3}$ \\ ${ }^{1}$ Hanoi Architectural University, Vietnam \\ ${ }^{2}$ Institute of Mechanics, VAST, Hanoi, Vietnam \\ ${ }^{3}$ University of Transport Technology, Hanoi, Vietnam \\ *E-mail: tunghv@hau.edu.vn
}

Received: 04 July 2020 / Published online: 27 September 2020

\begin{abstract}
For the first time, postbuckling behavior of thick doubly curved panels made of carbon nanotube reinforced composite (CNTRC), under preexisting external pressure and subjected to uniform temperature rise is analyzed in this paper. Carbon nanotubes (CNTs) are reinforced into matrix through functionally graded (FG) distribution patterns, and effective properties of CNTRC are determined according to extended rule of mixture. Formulations are based on a higher order shear deformation theory including Von Karman-Donnell nonlinearity, initial geometrical imperfection and elasticity of tangential constraints of boundary edges. Analytical solutions are assumed to satisfy simply supported boundary conditions and Galerkin method is used to obtain nonlinear load-deflection relation. Taking into account temperature dependence of material properties, postbuckling temperature-deflection paths are traced through an iteration process. The effects of preexisting external pressure, CNT volume fraction, tangential edge constraints, initial geometrical imperfection and curvature ratios on thermal postbuckling behavior of CNTRC doubly curved panels are analyzed through numerical examples. The study reveals that thermally loaded panels experiences a quasi-bifurcation response due to the presence of preexisting external pressure. For the most part, perfect panels are deflected toward convex side at the onset of undergoing thermal load. Particularly, imperfect panels may exhibit a bifurcation type buckling response when imperfection size satisfy a special condition.
\end{abstract}

Keywords: CNT-reinforced composite, thermal postbuckling response, higher order shear deformation theory, doubly curved panels, tangential edge constraints.

\section{INTRODUCTION}

Due to unprecedentedly excellent mechanical, thermal and electrical properties, carbon nanotubes (CNTs) have attracted huge attention of researchers of many fields [1]. These superior properties along with extremely large aspect ratio make CNTs become ideal fillers into isotropic matrix to form advanced nanocomposites. Motivated by the concept of functionally graded material (FGM), Shen [2] proposed functionally graded carbon nanotube reinforced composite (FG-CNTRC) in which CNTs are embedded into matrix in such a way that their volume fraction is varied in the thickness direction of the structure according to functional rules. Stimulated by Shen's proposal, numerous studies of static and dynamic responses of FG-CNTRC structures in general and FG-CNTRC curved panels and shells in particular have been performed. Buckling behavior of FG-CNTRC cylindrical panels under mechanical loads is analyzed in works of Macias et al. [3] and Zghal et al. [4] using finite element methods. Shen [5] studied the postbuckling of FG-CNTRC cylindrical panels under external pressure in thermal environments. Analytical investigations on thin and shear deformable FG-CNTRC cylindrical panels subjected to mechanical and thermomechanical loads are performed by Trang and Tung [6-8]. Postbuckling behavior 
of FG-CNTRC cylindrical shells subjected to axial compression and external pressure in thermal environments are analyzed by Shen $[9,10]$ employing higher order shear deformation theory (HSDT) and asymptotic solutions.

Since structural components are frequently exposed to severe temperature conditions, the stability of these components under thermal loads is a problem of considerable importance. Shen and Zhang [11] explored thermal buckling and postbuckling responses of higher order shear deformable FG-CNTRC plates subjected to two types of thermal load. Basing on first order shear deformation theory (FSDT) and Ritz method with Chebyshev shape functions, Kiani and coauthor [12,13] dealt with linear buckling problems of FG-CNTRC rectangular and skew plates under uniform temperature rise and various boundary conditions. Following a similar approach, Kiani $[14,15]$ also examined the postbuckling of FG-CNTRC plates and sandwich plates with FG-CNTRC face sheets under uniform temperature rise. Thermal buckling and postbuckling behaviors of thin and moderately thick FG-CNTRC plates have been treated in works $[16,17]$ using an analytical method. Long and Tung $[18,19]$ investigated thermal postbuckling of two sandwich plate models comprising isotropic and FG-CNTRC layers subjected to uniform temperature rise without and with preexisting axial compression. In these works, the FSDT, Galerkin method and an iteration procedure are utilized. Basing on different theories and approaches, thermal postbuckling analyses of FG-CNTRC cylindrical shells were carried out in works [20,21]. Using adjacent equilibrium criterion and a numerical solution, linear buckling problem of FG-CNTRC conical shells under uniform temperature rise has been treated by Mirzaei and Kiani [22]. Recently, Hieu and Tung [23] used an analytical approach and the FSDT to deal with linear buckling response of FG-CNTRC cylindrical shells and toroidal shell segments with elastically restrained edges.

The stability of curved panels under external pressure and thermal load is a crucial problem encountered in engineering applications. Postbuckling behavior of FG-CNTRC doubly curved panels with freely movable edges under external pressure is studied by Shen and Xiang [24] making use of HSDT, asymptotic solutions and a perturbation technique. Trang and Tung $[25,26]$ presented analytical investigations on the nonlinear stability of thin and first order shear deformable FG-CNTRC doubly curved panels under external pressure taking into account the effects of elastic foundations and tangential constraints of boundary edges. Thermal stability of composite and nanocomposite curved panels is a complicated problem. Unlike flat plate and circular cylindrical shells, due to curved configuration, membrane prebuckling state cannot exist. Previous studies [27-29] indicated that thermally loaded FGM curved panels with perfect geometry and immovable edges are monotonically deflected at the onset of heating. Linear and nonlinear buckling analyses of FG-CNTRC cylindrical panels under uniform temperature rise have been performed by Mehar et al. [30] and Shen and Xiang [31] employing numerical and semi-analytical approaches, respectively. Very recently, Trang and Tung [32] carried out a comprehensive analysis of possible types of thermal postbuckling response of higher order shear deformable FG-CNTRC cylindrical panels with initial imperfection and tangentially restrained edges. To the best of our knowledge, there is no investigation on thermal postbuckling behavior of FG-CNTRC doubly curved panels in the literature.

As an extension of previous work [32], the present paper aims to analyze the postbuckling behavior of thick FG-CNTRC doubly curved panels subjected to uniform temperature rise taking effects of preexisting external pressure into consideration. The properties of constituents are assumed to be temperature dependent, and effective properties of CNTRC are estimated by using extended rule of mixture. The panel is modelled within the framework of a higher order shear deformation theory including geometrical nonlinearity and initial imperfection. Analytical solutions are assumed to satisfy simply supported conditions of boundary edges and Galerkin method is applied to obtain nonlinear load-deflection relation. By adopting an iteration process, postbuckling paths are determined and interesting remarks are given. 


\section{FG-CNTRC DOUBLY CURVED PANELS}

This study considers shallow doubly curved panel with curved dimensions $a, b$ and thickness $h$ as shown in Fig. 1. The panel is defined in a coordinate system $x y z$ which the origin is located on the middle surface at one corner, $x$ and $y$ axes are directed to $a$ and $b$ dimensions, respectively, and $z$ is in the direction of inward normal to the middle surface. The curvature radii of the panel in $x$ and $y$ directions are denoted by $R_{x}$ and $R_{y}$, respectively. The panel is made of CNTRC and $x$ axis is the aligned direction of CNTs.

In this study, CNTs are reinforced into isotropic matrix through uniform distribution (UD) or three dif-

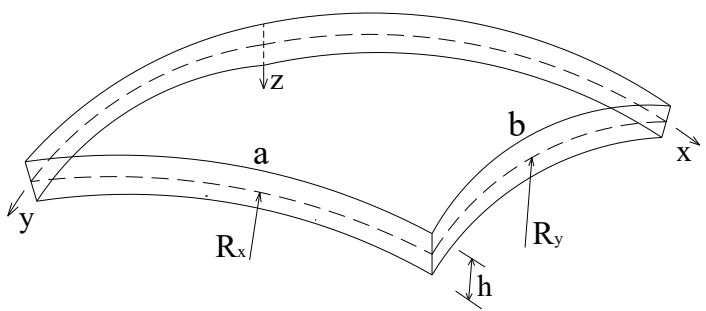

Fig. 1. Configuration and coordinate system of a doubly curved panel ferent types of functionally graded (FG) distributions, namely, FG-X, FG-V and FG- $\Lambda$ (Fig. 2). The volume fractions $V_{C N T}$ of CNTs corresponding to these distribution patterns are expressed as follows [2]

$$
V_{C N T}= \begin{cases}V_{C N T}^{*} & (\mathrm{UD}) \\ 2\left(\frac{2|z|}{h}\right) V_{C N T}^{*} & (\mathrm{FG}-\mathrm{X}) \\ \left(1-\frac{2 z}{h}\right) V_{C N T}^{*} & (\mathrm{FG}-\mathrm{V}) \\ \left(1+\frac{2 z}{h}\right) V_{C N T}^{*} & (\mathrm{FG}-\Lambda)\end{cases}
$$

in which $V_{C N T}^{*}$ is total volume fraction of CNTs and its specific expression can be found in many previous works, e.g. $[2,11]$.

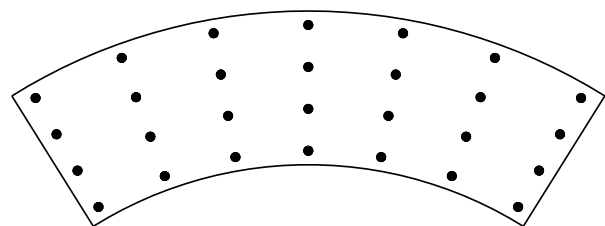

UD

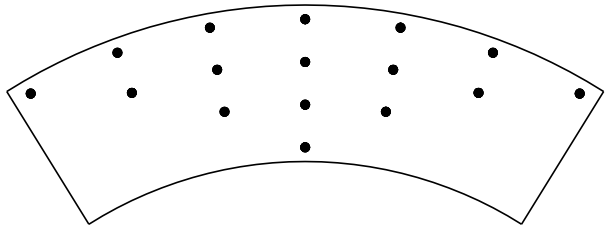

FG-V

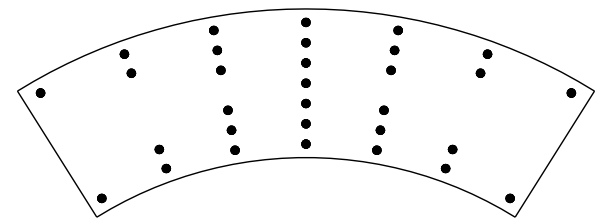

FG-X

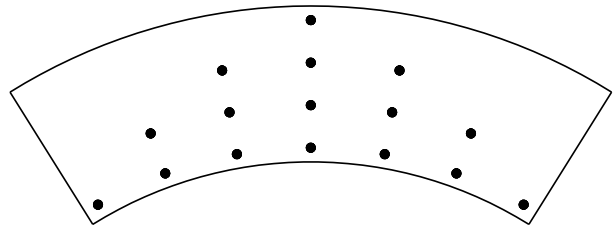

FG- $\Lambda$

Fig. 2. Different types of CNT distribution

In this study, effective elastic moduli $E_{11}, E_{22}$ and effective shear modulus $G_{12}$ are determined according to extended rule of mixture as [2]

$$
\begin{aligned}
& E_{11}=\eta_{1} V_{C N T} E_{11}^{C N T}+V_{m} E^{m}, \\
& \frac{\eta_{2}}{E_{22}}=\frac{V_{C N T}}{E_{22}^{C N T}}+\frac{V_{m}}{E^{m}}, \\
& \frac{\eta_{3}}{G_{12}}=\frac{V_{C N T}}{G_{12}^{C N T}}+\frac{V_{m}}{G^{m}},
\end{aligned}
$$


in which $\eta_{1}, \eta_{2}$, and $\eta_{3}$ are CNT efficiency parameters, $E_{11}^{C N T}, E_{22}^{C N T}$ and $G_{12}^{C N T}$ are elastic moduli and shear modulus of CNTs, respectively, whereas $V_{m}=1-V_{C N T}, E^{m}$ and $G^{m}$ denote the volume fraction, modulus of elasticity and shear modulus of matrix, respectively. In addition, it is assumed that effective shear moduli $G_{13}=G_{12}$ and $G_{23}=1.2 G_{12}[9,11]$

Due to weak dependence on position and temperature, effective Poisson ratio is assumed to be constant and determined according to linear rule of mixture as follows

$$
v_{12}=V_{C N T}^{*} v_{12}^{C N T}+\left(1-V_{C N T}^{*}\right) v^{m},
$$

where $v_{12}^{\mathrm{CNT}}$ and $v^{m}$ are Poisson ratios of CNTs and matrix, respectively.

Effective thermal expansion coefficients $\alpha_{11}$ and $\alpha_{22}$ of CNTRC in longitudinal and transverse directions, respectively, are evaluated based on Schapery model as $[12,20]$

$$
\begin{aligned}
\alpha_{11} & =\frac{V_{C N T} E_{11}^{C N T} \alpha_{11}^{C N T}+V_{m} E^{m} \alpha^{m}}{V_{C N T} E_{11}^{C N T}+V_{m} E^{m}}, \\
\alpha_{22} & =\left(1+v_{12}^{C N T}\right) V_{C N T} \alpha_{22}^{C N T}+\left(1+v^{m}\right) V_{m} \alpha^{m}-v_{12} \alpha_{11},
\end{aligned}
$$

where $\alpha_{11}^{C N T}, \alpha_{22}^{C N T}$ and $\alpha^{m}$ denote thermal expansion coefficients of CNTs and matrix, respectively.

\section{FORMULATIONS}

In the present work, mathematical formulations are established within the framework of higher order shear shell theory (HSDT) developed by Reddy and Liu [33]. Based on the HSDT, in-plane strain components $\varepsilon_{x}, \varepsilon_{y}, \gamma_{x y}$ and transverse shear deformations $\gamma_{x z}, \gamma_{y z}$ at a $z$ distance from the middle surface are expressed as the following

$$
\left\{\begin{array}{c}
\varepsilon_{x} \\
\varepsilon_{y} \\
\gamma_{x y}
\end{array}\right\}=\left\{\begin{array}{c}
\varepsilon_{x}^{0} \\
\varepsilon_{y}^{0} \\
\gamma_{x y}^{0}
\end{array}\right\}+z\left\{\begin{array}{c}
k_{x}^{1} \\
k_{y}^{1} \\
k_{x y}^{1}
\end{array}\right\}+z^{3}\left\{\begin{array}{c}
k_{x}^{3} \\
k_{y}^{3} \\
k_{x y}^{3}
\end{array}\right\},\left\{\begin{array}{c}
\gamma_{x z} \\
\gamma_{y z}
\end{array}\right\}=\left\{\begin{array}{c}
\gamma_{x z}^{0} \\
\gamma_{y z}^{0}
\end{array}\right\}+z^{2}\left\{\begin{array}{c}
k_{x z}^{2} \\
k_{y z}^{2}
\end{array}\right\}
$$

where

$$
\begin{gathered}
\left\{\begin{array}{c}
\varepsilon_{x}^{0} \\
\varepsilon_{y}^{0} \\
\gamma_{x y}^{0}
\end{array}\right\}=\left\{\begin{array}{c}
u, x-\frac{w}{R_{x}}+\frac{1}{2} w_{, x}^{2} \\
v_{, y}-\frac{w}{R_{y}}+\frac{1}{2} w_{, y}^{2} \\
u_{, y}+v_{, x}+w_{, x} w_{, y}
\end{array}\right\},\left\{\begin{array}{c}
k_{x}^{1} \\
k_{y}^{1} \\
k_{x y}^{1}
\end{array}\right\}=\left\{\begin{array}{c}
\phi_{x, x} \\
\phi_{y, y} \\
\phi_{x, y}+\phi_{y, x}
\end{array}\right\}, \\
\left\{\begin{array}{c}
k_{x}^{3} \\
k_{y}^{3} \\
k_{x y}^{3}
\end{array}\right\}=-c\left\{\begin{array}{c}
\phi_{x, x}+w_{, x x} \\
\phi_{y, y}+w_{, y y} \\
\phi_{x, y}+\phi_{y, x}+2 w_{, x y}
\end{array}\right\},\left\{\begin{array}{l}
\gamma_{x z}^{0} \\
\gamma_{y z}^{0}
\end{array}\right\}=\left\{\begin{array}{l}
\phi_{x}+w_{, x} \\
\phi_{y}+w_{, y}
\end{array}\right\},\left\{\begin{array}{l}
k_{x z}^{2} \\
k_{y z}^{2}
\end{array}\right\}=-3 c\left\{\begin{array}{l}
\phi_{x}+w_{, x} \\
\phi_{y}+w_{, y}
\end{array}\right\},
\end{gathered}
$$

in which $c=4 /\left(3 h^{2}\right), u, v$ and $w$ are in-plane displacements and lateral displacement (i.e. deflection), respectively, whereas $\phi_{x}$ and $\phi_{y}$ are rotations of a normal to the middle surface with respect to $y$ and $x$ axes, respectively. Herein, subscript comma indicates partial derivative with respect to the followed variable, e.g. $u, x=\partial u / \partial x$.

In this study, the panel is exposed to elevated temperature $T$ and stress components are determined according to constitutive relations as

where

$$
\left(\begin{array}{c}
\sigma_{x} \\
\sigma_{y} \\
\sigma_{x y} \\
\sigma_{x z} \\
\sigma_{y z}
\end{array}\right)=\left(\begin{array}{ccccc}
Q_{11} & Q_{12} & 0 & 0 & 0 \\
Q_{12} & Q_{22} & 0 & 0 & 0 \\
0 & 0 & Q_{66} & 0 & 0 \\
0 & 0 & 0 & Q_{44} & 0 \\
0 & 0 & 0 & 0 & Q_{55}
\end{array}\right)\left(\begin{array}{c}
\varepsilon_{x}-\alpha_{11} \Delta T \\
\varepsilon_{y}-\alpha_{22} \Delta T \\
\gamma_{x y} \\
\gamma_{x z} \\
\gamma_{y z}
\end{array}\right),
$$

$$
Q_{11}=\frac{E_{11}}{1-v_{12} v_{21}}, Q_{22}=\frac{E_{22}}{1-v_{12} v_{21}}, Q_{12}=\frac{v_{21} E_{11}}{1-v_{12} v_{21}}, Q_{44}=G_{13}, Q_{55}=G_{23}, Q_{66}=G_{12},
$$


and $\Delta T=T-T_{0}$ is uniform temperature rise from initial temperature $T_{0}$ at which the panel is free from thermal stresses.

Force and moment resultants per a unit length are calculated through stress components as

$$
\begin{aligned}
& \left(N_{x}, N_{y}, N_{x y}\right)=\int_{-h / 2}^{h / 2}\left(\sigma_{x}, \sigma_{y}, \sigma_{x y}\right) \mathrm{d} z, \quad\left(Q_{x}, Q_{y}\right)=\int_{-h / 2}^{h / 2}\left(\sigma_{x z}, \sigma_{y z}\right) \mathrm{d} z, \\
& \left(H_{x}, H_{y}\right)=\int_{-h / 2}^{h / 2}\left(\sigma_{x z}, \sigma_{y z}\right) z^{2} \mathrm{~d} z, \quad\left(M_{x}, M_{y}, M_{x y}\right)=\int_{-h / 2}^{h / 2}\left(\sigma_{x}, \sigma_{y}, \sigma_{x y}\right) z \mathrm{~d} z, \\
& \left(P_{x}, P_{y}, P_{x y}\right)=\int_{-h / 2}^{h / 2}\left(\sigma_{x}, \sigma_{y}, \sigma_{x y}\right) z^{3} \mathrm{~d} z,
\end{aligned}
$$

and, from Eqs. (5) and (7), these resultants are expressed in the form

$$
\begin{gathered}
{\left[\begin{array}{c}
N_{x} \\
N_{y} \\
N_{x y} \\
M_{x} \\
M_{y} \\
M_{x y} \\
P_{x} \\
P_{y} \\
P_{x y}
\end{array}\right]=\left[\begin{array}{ccccccccc}
e_{11} & v_{21} e_{11} & 0 & e_{12} & v_{21} e_{12} & 0 & e_{14} & v_{21} e_{14} & 0 \\
v_{12} e_{21} & e_{21} & 0 & v_{12} e_{22} & e_{22} & 0 & v_{12} e_{24} & e_{24} & 0 \\
0 & 0 & e_{31} & 0 & 0 & e_{32} & 0 & 0 & e_{34} \\
e_{12} & v_{21} e_{12} & 0 & e_{13} & v_{21} e_{13} & 0 & e_{15} & v_{21} e_{15} & 0 \\
v_{12} e_{22} & e_{22} & 0 & v_{12} e_{23} & e_{23} & 0 & v_{12} e_{25} & e_{25} & 0 \\
0 & 0 & e_{32} & 0 & 0 & e_{33} & 0 & 0 & e_{35} \\
e_{14} & v_{21} e_{14} & 0 & e_{15} & v_{21} e_{15} & 0 & e_{17} & v_{21} e_{17} & 0 \\
v_{12} e_{24} & e_{24} & 0 & v_{12} e_{25} & e_{25} & 0 & v_{12} e_{27} & e_{27} & 0 \\
0 & 0 & e_{34} & 0 & 0 & e_{35} & 0 & 0 & e_{37}
\end{array}\right]\left[\begin{array}{c}
\varepsilon_{x}^{0} \\
\varepsilon_{y}^{0} \\
\gamma_{x y}^{0} \\
k_{x}^{1} \\
k_{y}^{1} \\
k_{x y}^{1} \\
k_{x}^{3} \\
k_{y}^{3} \\
k_{x y}^{3}
\end{array}\right]-\left[\begin{array}{c}
e_{11 T} \\
e_{21 T} \\
0 \\
e_{12 T} \\
e_{22 T} \\
0 \\
e_{14 T} \\
e_{24 T} \\
0
\end{array}\right]} \\
{\left[\begin{array}{c}
Q_{x} \\
Q_{y} \\
H_{x} \\
H_{y}
\end{array}\right]=\left[\begin{array}{cccc}
e_{41} & 0 & e_{43} & 0 \\
0 & e_{51} & 0 & e_{53} \\
e_{43} & 0 & e_{45} & 0 \\
0 & e_{53} & 0 & e_{55}
\end{array}\right]\left[\begin{array}{c}
\gamma_{x z}^{0} \\
\gamma_{y z}^{0} \\
k_{x z}^{2} \\
k_{y z}^{2}
\end{array}\right],}
\end{gathered}
$$

where the detailed definitions of components $e_{i j}$ and $e_{k l T}$ can be found in the work [32].

Based on the HSDT, system of five nonlinear equilibrium equations of geometrically perfect doubly curved panels is expressed as follows [33]

$$
\begin{gathered}
N_{x, x}+N_{x y, y}=0, \\
N_{x y, x}+N_{y, y}=0, \\
Q_{x, x}+Q_{y, y}-3 c\left(H_{x, x}+H_{y, y}\right)+c\left(P_{x, x x}+2 P_{x y, x y}+P_{y, y y}\right) \\
+N_{x} w_{, x x}+2 N_{x y} w_{, x y}+N_{y} w_{, y y}+\frac{N_{x}}{R_{x}}+\frac{N_{y}}{R_{y}}+q=0, \\
M_{x, x}+M_{x y, y}-Q_{x}+3 c H_{x}-c\left(P_{x, x}+P_{x y, y}\right)=0, \\
M_{x y, x}+M_{y, y}-Q_{y}+3 c H_{y}-c\left(P_{x y, x}+P_{y, y}\right)=0,
\end{gathered}
$$

where $q$ is external pressure uniformly distributed on the top surface of the panel.

By introducing a stress function $f(x, y)$ defined as $N_{x}=f_{, y y}, N_{y}=f_{, x x}, N_{x y}=-f_{, x y}$ and following mathematical transformations as described in previous works [26,27], nonlinear equilibrium equation of geometrically imperfect FG-CNTRC doubly curved panels is written in the form

$$
\begin{aligned}
& a_{11} \phi_{x, x x x}+a_{21} \phi_{x, x y y}+a_{31} \phi_{y, x x y}+a_{41} \phi_{y, y y y}+a_{51} f_{, x x y y}+a_{61} w_{, x x x x}+a_{71} w_{, x x y y}+a_{81} w_{, y y y y} \\
& +f_{, y y}\left(w_{, x x}+w_{, x x}^{*}\right)-2 f_{, x y}\left(w_{, x y}+w_{, x y}^{*}\right)+f_{, x x}\left(w_{, y y}+w_{, y y}^{*}\right)+\frac{f_{, y y}}{R_{x}}+\frac{f_{, x x}}{R_{y}}+q=0,
\end{aligned}
$$

where $w^{*}(x, y)$ is a known function representing initial geometrical imperfection and coefficients $a_{i 1}$ $(i=1 \div 8)$ are given in the work [32]. 
From Eq. (6), strain compatibility equation of a doubly curved panel has the form

$$
\varepsilon_{x, y y}^{0}+\varepsilon_{y, x x}^{0}-\gamma_{x y, x y}^{0}=w_{, x y}^{2}-w_{, x x} w_{, y y}-\frac{w_{, x x}}{R_{y}}-\frac{w_{, y y}}{R_{x}} .
$$

By solving Eq. (10) for $\varepsilon_{x}^{0}, \varepsilon_{y}^{0}, \gamma_{x y}^{0}$ and including initial imperfection, Eq. (14) can be rewritten in the following form

$$
\begin{aligned}
& a_{12} f_{, x x x x}+a_{22} f_{, x x y y}+a_{32} f_{, y y y y}+a_{42} \phi_{x, x x x}+a_{52} \phi_{y, x x y}+a_{62} \phi_{y, y y y}+a_{72} \phi_{x, x y y}+a_{82} w_{, x x x x} \\
& +a_{92} w_{, x x y y}+a_{02} w_{, y y y y}-w_{, x y}^{2}+w_{, x x} w_{, y y}-2 w_{, x y} w_{, x y}^{*}+w_{, x x} w_{, y y}^{*}+w_{, y y} w_{, x x}^{*}+\frac{w_{, x x}}{R_{y}}+\frac{w_{, y y}}{R_{x}}=0,
\end{aligned}
$$

in which coefficients $a_{j 2}(j=0 \div 9)$ can be found in the work [32].

In the present work, all edges of panel are assumed to be simply supported and elastically restrained in tangential displacements. The associated boundary conditions are expressed as

$$
\begin{aligned}
& w=N_{x y}=\phi_{y}=M_{x}=P_{x}=0, N_{x}=N_{x 0} \text { at } x=0, a \\
& w=N_{x y}=\phi_{x}=M_{y}=P_{y}=0, N_{y}=N_{y 0} \text { at } y=0, b
\end{aligned}
$$

in which $N_{x 0}$ and $N_{y 0}$ are fictitious compressive force resultants at edges $x=0, a$ and $y=0, b$, respectively, and related to average end-shortening displacements as follows [26,32]

$$
N_{x 0}=-\frac{c_{1}}{a b} \int_{0}^{a} \int_{0}^{b} \frac{\partial u}{\partial x} \mathrm{~d} y \mathrm{~d} x, \quad N_{y 0}=-\frac{c_{2}}{a b} \int_{0}^{a} \int_{0}^{b} \frac{\partial v}{\partial y} \mathrm{~d} y \mathrm{~d} x,
$$

where $c_{1}$ and $c_{2}$ are average tangential stiffness parameters at opposite edges $x=0, a$ and $y=0, b$, respectively.

To satisfy boundary conditions (16) approximately, the following analytical solutions are assumed

$$
\begin{gathered}
w=W \sin \beta_{m} x \sin \delta_{n} y, \quad w^{*}=\mu h \sin \beta_{m} x \sin \delta_{n} y, \\
f=A_{1} \cos 2 \beta_{m} x+A_{2} \cos 2 \delta_{n} y+A_{3} \sin \beta_{m} x \sin \delta_{n} y+\frac{1}{2} N_{x 0} y^{2}+\frac{1}{2} N_{y 0} x^{2}, \\
\phi_{x}=B_{1} \cos \beta_{m} x \sin \delta_{n} y, \quad \phi_{y}=B_{2} \sin \beta_{m} x \cos \delta_{n} y,
\end{gathered}
$$

where $\beta_{m}=m \pi / a, \delta_{n}=n \pi / b(m, n=1,2, \ldots), W$ is amplitude of the deflection and $\mu$ is size of imperfection. In addition, in the Eqs. (18), $A_{i}(i=1 \div 3)$ and $B_{j}(j=1,2)$ are coefficients to be determined.

Introduction of Eqs. (18a)-(18b) into compatibility equation (15) gives the results

$$
\begin{aligned}
A_{1}=\frac{\delta_{n}^{2}}{32 a_{12} \beta_{m}^{2}}\left(W^{2}+\right. & 2 W \mu h), \quad A_{2}=\frac{\beta_{m}^{2}}{32 a_{32} \delta_{n}^{2}}\left(W^{2}+2 W \mu h\right) \\
\left(a_{12} \beta_{m}^{4}+a_{22} \beta_{m}^{2} \delta_{n}^{2}+a_{32} \delta_{n}^{4}\right) A_{3} & +\left(a_{42} \beta_{m}^{3}+a_{72} \beta_{m} \delta_{n}^{2}\right) B_{1}+\left(a_{52} \beta_{m}^{2} \delta_{n}+a_{62} \delta_{n}^{3}\right) B_{2} \\
& +\left(a_{82} \beta_{m}^{4}+a_{92} \beta_{m}^{2} \delta_{n}^{2}+a_{02} \delta_{n}^{4}-\frac{\delta_{n}^{2}}{R_{x}}-\frac{\beta_{m}^{2}}{R_{y}}\right) W=0 .
\end{aligned}
$$

Subsequently, substituting the Eqs. (6) and (10) into the last two equilibrium equations (12d)-(12e) and putting the solutions (18a), (18c) into the obtained partial differential equations, we receive a system of two algebraic equations in terms of $A_{3}, B_{1}$ and $B_{2}$. Then, solving two these equations in combination with Eq. (19b) yields the following coefficients

$$
A_{3}=A_{3}^{*} W, \quad B_{1}=B_{1}^{*} W, \quad B_{2}=B_{2}^{*} W,
$$

where

$$
\begin{gathered}
A_{3}^{*}=\frac{1}{b_{14}}\left[\left(\frac{\delta_{n}^{2}}{R_{x}}+\frac{\beta_{m}^{2}}{R_{y}}\right) b_{24}-b_{34}\right], \quad B_{1}^{*}=\frac{b_{13} b_{32}-b_{12} b_{33}}{b_{22} b_{33}-b_{23} b_{32}} A_{3}^{*}+\frac{b_{33} b_{42}-b_{32} b_{43}}{b_{22} b_{33}-b_{23} b_{32}}, \\
B_{2}^{*}=\frac{b_{12} b_{23}-b_{13} b_{22}}{b_{22} b_{33}-b_{23} b_{32}} A_{3}^{*}+\frac{b_{22} b_{43}-b_{23} b_{42}}{b_{22} b_{33}-b_{23} b_{32}},
\end{gathered}
$$

in which $b_{i j}(i=1 \div 4, j=1 \div 4)$ are given in the work [32]. 
Now, introducing the solutions (18) into the equilibrium equation (13) and applying Galerkin method to the resulting equation, we obtain

$$
\begin{aligned}
& a_{13} \bar{W}+a_{23} \bar{W}(\bar{W}+\mu)+a_{33} \bar{W}(\bar{W}+2 \mu)+a_{43} \bar{W}(\bar{W}+\mu)(\bar{W}+2 \mu) \\
& -\left(\bar{N}_{x 0} m^{2} B_{a}^{2}+\bar{N}_{y 0} n^{2}\right) \frac{\pi^{2}}{B_{h}^{2}}(\bar{W}+\mu)+\left(\bar{N}_{x 0} B_{a} R_{a x}+\bar{N}_{y 0} R_{b y}\right) \frac{16 \gamma_{m} \gamma_{n}}{m n \pi^{2} B_{h}}+\frac{16 \gamma_{m} \gamma_{n}}{m n \pi^{2}} q=0,
\end{aligned}
$$

where

$$
\begin{aligned}
& B_{a}=\frac{b}{a}, \quad B_{h}=\frac{b}{h}, \quad R_{a x}=\frac{a}{R_{x}}, \quad R_{b y}=\frac{b}{R_{y}}, \\
& \left(\bar{N}_{x 0}, \bar{N}_{y 0}, \bar{W}\right)=\frac{1}{h}\left(N_{x 0}, N_{y 0}, W\right), \quad \gamma_{k}=\frac{1}{2}\left[1-(-1)^{k}\right], \quad(k=m, n)
\end{aligned}
$$

and coefficients $a_{k 3}(k=1 \div 4)$ are displayed in Eq. (A1) in Appendix A.

In what follows, fictitious force resultants $\bar{N}_{x 0}$ and $\bar{N}_{y 0}$ will be determined. From Eqs. (6) and (10), the expressions of $u_{, x}$ and $v, y$ can be obtained. Afterwards, substituting the solutions (18) into the $u_{, x}$ and $v, y$ and placing the received expressions into Eq. (17) lead to the following expressions

$$
\begin{aligned}
& \bar{N}_{x 0}=a_{16} \bar{W}+a_{26} \bar{W}(\bar{W}+2 \mu)+a_{36} \Delta T, \\
& \bar{N}_{y 0}=a_{17} \bar{W}+a_{27} \bar{W}(\bar{W}+2 \mu)+a_{37} \Delta T,
\end{aligned}
$$

where the detailed definitions of coefficients $a_{i 6}$ and $a_{i 7}(i=1 \div 3)$ are given in Eq. (B1) in Appendix B.

Now, introduction of the Eqs. (24) into the Eq. (22) gives the following relation

$$
\Delta T=\frac{1}{a_{58}}\left[a_{18} \bar{W}+a_{28} \bar{W}(\bar{W}+\mu)+a_{38} \bar{W}(\bar{W}+2 \mu)+a_{48} \bar{W}(\bar{W}+\mu)(\bar{W}+2 \mu)+\frac{16 \gamma_{m} \gamma_{n}}{m n \pi^{2}} q\right],
$$

where

$$
\begin{aligned}
& a_{18}=a_{13}+\frac{16 \gamma_{m} \gamma_{n}}{m n \pi^{2} B_{h}}\left(a_{16} B_{a} R_{a x}+a_{17} R_{b y}\right), \quad a_{28}=a_{23}-a_{16} m^{2} \pi^{2} \frac{B_{a}^{2}}{B_{h}^{2}}-a_{17} \frac{n^{2} \pi^{2}}{B_{h}^{2}}, \\
& a_{38}=a_{33}+\frac{16 \gamma_{m} \gamma_{n}}{m n \pi^{2} B_{h}}\left(a_{26} B_{a} R_{a x}+a_{27} R_{b y}\right), \quad a_{48}=a_{43}-a_{26} B_{a}^{2} \frac{m^{2} \pi^{2}}{B_{h}^{2}}-a_{27} \frac{n^{2} \pi^{2}}{B_{h}^{2}}, \\
& a_{58}=\left(a_{36} B_{a}^{2} \frac{m^{2} \pi^{2}}{B_{h}^{2}}+a_{37} \frac{n^{2} \pi^{2}}{B_{h}^{2}}\right)(\bar{W}+\mu)-\frac{16 \gamma_{m} \gamma_{n}}{m n \pi^{2} B_{h}}\left(a_{36} B_{a} R_{a x}+a_{37} R_{b y}\right) .
\end{aligned}
$$

Eq. (25) expresses nonlinear load-deflection relation of FG-CNTRC doubly curved panels under preexisting external pressure and subjected to uniform temperature rise. It is recognized from Eqs. (25) and (26) that if $q=0$ the thermally loaded panels will be deflected at the onset of heating and, in general, no bifurcation buckling occurs. Especially, bifurcation buckling response may occurs for imperfect panels when imperfection size $\mu$ satisfies a special condition predicted from Eq. (26) as follows

$$
\mu=\mu_{b}=16 \gamma_{m} \gamma_{n} B_{h} \frac{a_{36} B_{a} R_{a x}+a_{37} R_{b y}}{m n \pi^{4}\left(a_{36} m^{2} B_{a}^{2}+a_{37} n^{2}\right)} .
$$

It is obvious from Eq. (27) that $\mu_{b}=0$ when $R_{a x}=R_{b y}=0$. This implies that, as expected, perfectly flat plate will be buckled in bifurcation type under thermal loads.

Due to temperature dependence of material properties, temperature-deflection paths will be determined through an iteration process.

\section{RESULTS AND DISCUSSION}

This section graphically presents numerical results for thermal postbuckling analysis of shallow doubly curved panels with square planform $(a=b)$ made of Poly (methyl methacrylate) matrix material, referred to as PMMA, and reinforced by $(10,10)$ single-walled carbon nanotubes (SWCNTs). Temperature dependent properties of PMMA and SWCNTs have been given in many previous works, for examples $[5,9,12,20]$, and omitted here for the sake of brevity. In numerical results, CNT efficiency parameters are chosen as those given in the works $[5,9,11]$, specifically, $\left(\eta_{1}, \eta_{2}, \eta_{3}\right)=(0.137,1.022,0.715)$ for the case of $V_{C N T}^{*}=0.12,\left(\eta_{1}, \eta_{2}, \eta_{3}\right)=(0.142,1.626,1.138)$ for the case of $V_{C N T}^{*}=0.17$, and $\left(\eta_{1}, \eta_{2}, \eta_{3}\right)=$ 
$(0.141,1.585,1.109)$ for the case of $V_{C N T}^{*}=0.28$. To measure degree of tangential edge constraints more conveniently, non-dimensional tangential stiffness parameters are defined as follows $[16,17,26,32]$

$$
\lambda_{1}=\frac{c_{1}}{e_{11}+c_{1}}, \quad \lambda_{2}=\frac{c_{2}}{e_{11}+c_{2}} .
$$

According to this definition, movable $\left(c_{1}=0\right)$, immovable $\left(c_{1} \rightarrow \infty\right)$ and partially movable $\left(0<c_{1}<\infty\right)$ edges $x=0, a$ are characterized by $\lambda_{1}=0, \lambda_{1}=1$ and $0<\lambda_{1}<1$, respectively. Similarly, values of $\lambda_{2}=0$ (i.e. $c_{2}=0$ ), $\lambda_{2}=1$ (i.e. $c_{2} \rightarrow \infty$ ) and $0<\lambda_{2}<1$ (i.e. $0<c_{2}<\infty$ ) represent movable, immovable and partially movable edges $y=0, b$, respectively. For the sake of brief expressions, CNTRC doubly curved panels are assumed to be geometrically perfect $(\mu=0)$, with immovable edges $\left(\lambda_{1}=\lambda_{2}=1\right)$, and with temperature dependent properties, unless otherwise specified. Furthermore, temperature independent and temperature dependent properties will be referred to here as T-ID and T-D properties, respectively, and T-ID properties are those evaluated at room temperature $\left(T_{0}=300 \mathrm{~K}\right)$.

There is no investigation on thermal postbuckling of FG-CNTRC doubly curved panels in the literature for direct comparison. Comparative studies for particular cases of panel geometry, namely, flat panel $\left(R_{x} \rightarrow \infty, R_{y} \rightarrow \infty\right)$ and cylindrical panel $\left(R_{x} \rightarrow \infty, R_{y}<\infty\right)$, have been performed in previous work [32]. The mentioned comparisons achieved good agreements and verified the proposed approach.

\subsection{Thermal postbuckling of panels without external pressure}

Numerical results for thermal postbuckling behavior of CNTRC doubly curved panels only subjected to uniform temperature rise are shown in Figs. 3-8. The effects of CNT distribution on thermal postbuckling of shallow CNTRC panels are depicted in Fig. 3. Unlike flat panels, due to curved configuration, thermally loaded doubly curved panels have no prebuckling membrane state and are deflected towards convex side (i.e. negative deflection) at the onset of heating. Generally, among three types of CNT reinforcement, FG-X and FG- $\Lambda$ panels have the strongest and weakest load carrying capacities, respectively, in small region of deflection. Particularly, in the deep region of deflection, load-deflection path of FG-V panel is slightly higher than that of FG-X panel. In the remainder of numerical examples, only panels with FG-X type of CNT distribution are considered.

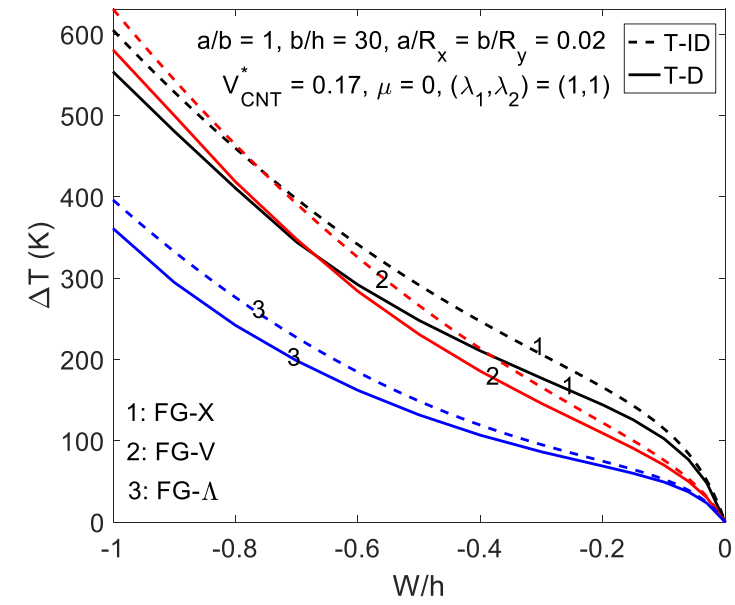

Fig. 3. Effects of CNT distribution patterns on thermal postbuckling response of FG-CNTRC doubly curved panels with immovable edges

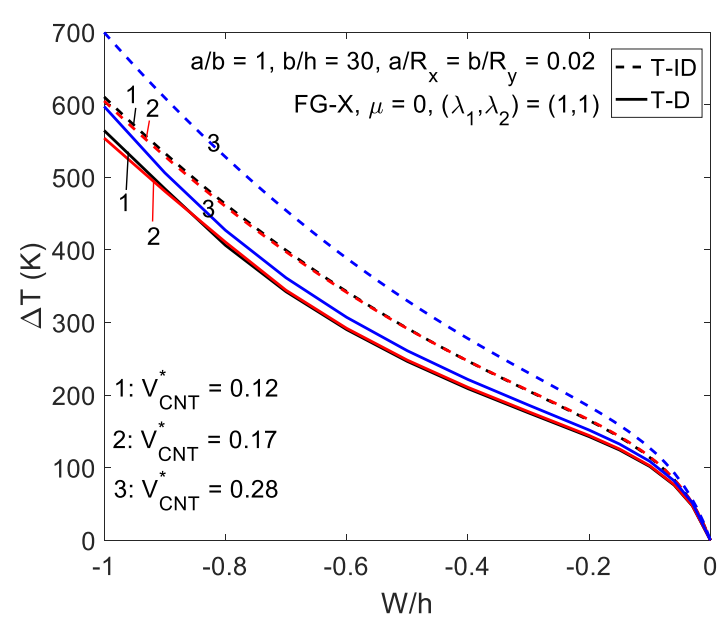

Fig. 4. Effects of CNT volume fraction on thermal postbuckling response of FG-CNTRC doubly curved panels with immovable edges

Next, Fig. 4 assesses the effects of total volume fraction $V_{C N T}^{*}$ of CNTs on thermal postbuckling of FG-CNTRC panels. As shown, postbuckling path corresponding to $V_{C N T}^{*}=0.28$ is the highest, while postbuckling paths corresponding to $V_{C N T}^{*}=0.12$ and $V_{C N T}^{*}=0.17$ are almost coincided. Figs. 3 and 4 also demonstrate that load carrying capability of CNTRC panels are pronouncedly dropped when 
temperature dependence of material properties are taken into consideration. The effects of curvature on thermal postbuckling response of FG-CNTRC panels are shown in Fig. 5 plotted with five different pairs of $\left(a / R_{x}, b / R_{y}\right)$ ratios. While flat panel (i.e. $\left.a / R_{x}=b / R_{y}=0\right)$ exhibits a bifurcation type buckling response and a symmetric postbuckling path, curved panels have no bifurcation buckling response when edges are immovable and geometry is perfect. Moreover, in the deep region of deflection (i.e. large deflection region), more curved panels (i.e. larger values of $\left(a / R_{x}, b / R_{y}\right)$ ratios) have higher equilibrium paths.

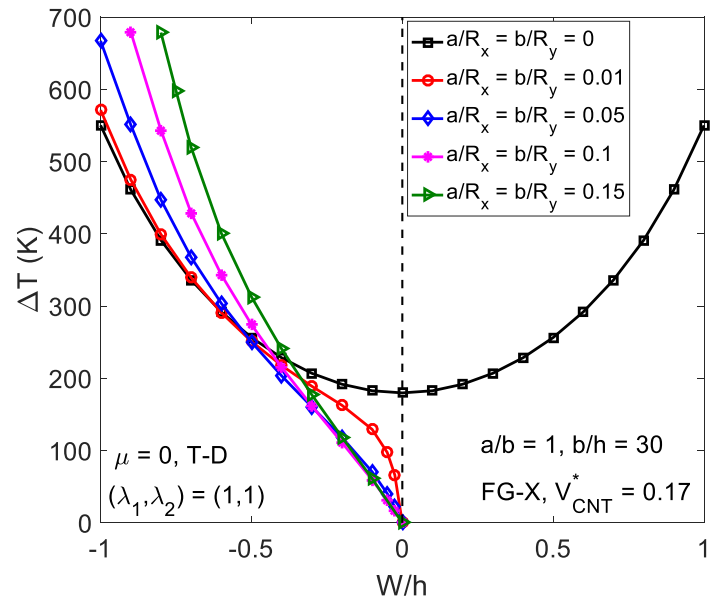

Fig. 5. Effects of curvature on thermal postbuckling response of FG-CNTRC doubly curved panels with immovable edges

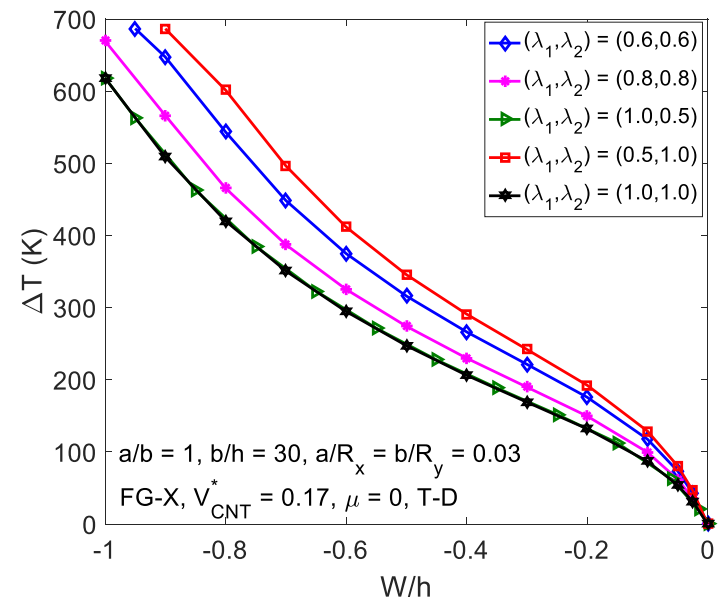

Fig. 6. Effects of tangential edge constraints on thermal postbuckling response of FG-CNTRC doubly curved panels

As a subsequent illustration, the effects of various degrees of tangential edge constraints on thermal postbuckling of FG-CNTRC doubly curved panels are analyzed in Fig. 6 plotted with six different pairs of $\left(\lambda_{1}, \lambda_{2}\right)$ parameters. It is clear that postbuckling strength of panels is significantly reduced when $\left(\lambda_{1}, \lambda_{2}\right)$ parameters become larger, i.e., edges are more severely restrained. In addition, it is recognized from Fig. 6 that constraints of $x=0, a$ and $y=0, b$ edges have remarkable and slight influences on postbuckling response of the panel, respectively. This fact reflects the high anisotropy of CNT which its longitudinal elastic modulus $E_{11}^{C N T}$ is much higher than transverse elastic modulus $E_{22}^{C N T}$.

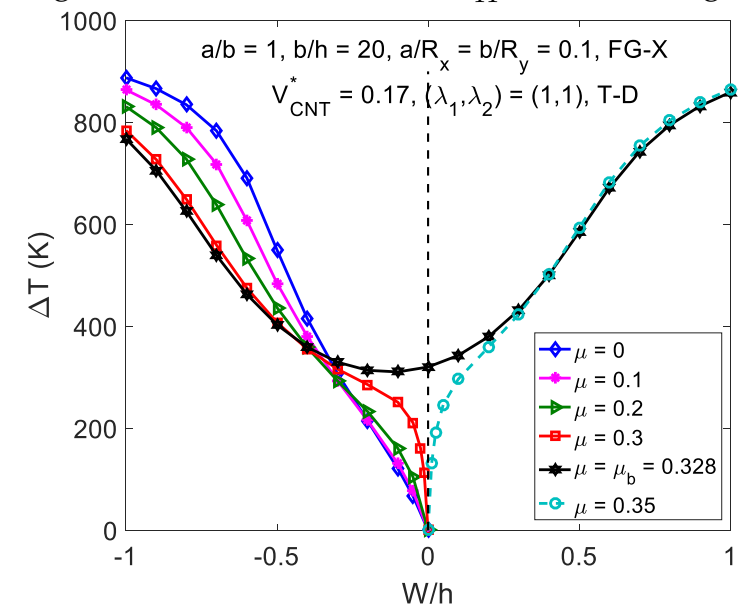

Fig. 7. Effects of initial geometrical imperfection on thermal postbuckling response of FG-CNTRC doubly curved panels

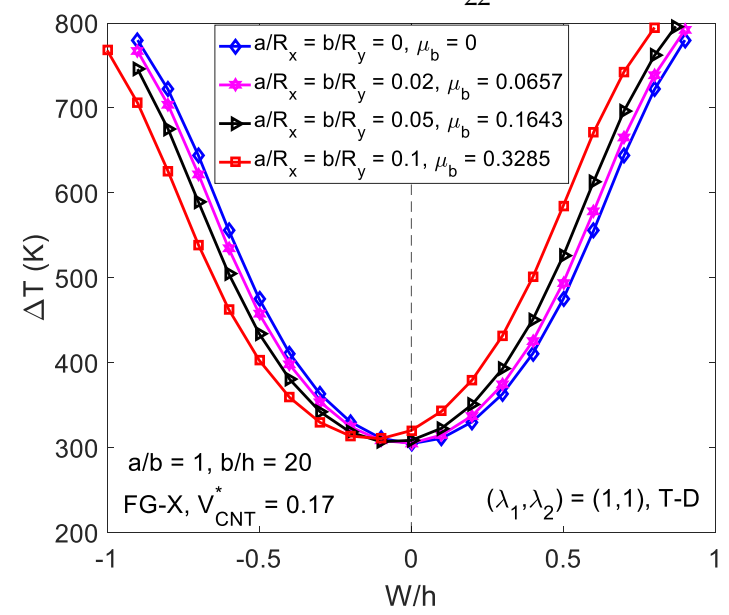

Fig. 8. Interactive effects of curvature and imperfection on thermal postbuckling response of FG-CNTRC doubly curved panels

To examine the effects of initial geometrical imperfection on thermal postbuckling response of FGCNTRC doubly curved panels, Fig. 7 depicts postbuckling paths corresponding to six different values 
of imperfection size $\mu$. As can be observed, for smaller values of $\mu$, the panel is deflected towards convex side, and temperature-deflection paths are higher and lower in small and larger regions of negative deflection when $\mu$ is increased, respectively. When $\mu$ reaches a special value predicted by Eq. (27) $\left(\mu=\mu_{b}=0.328\right.$ for this specific example), the panel exhibits a bifurcation type buckling response with asymmetric postbuckling path. As $\mu$ exceeds special value $\mu_{b}$, the panel will be deflected toward concave side (i.e. positive deflection) at the onset of applying thermal load. Next example is shown in Fig. 8 considering interactive effects of curvature and initial imperfection on bifurcation buckling and thermal postbuckling responses of geometrically imperfect FG-CNTRC doubly curved panels. Obviously, more curved panels may be buckled in bifurcation type when imperfection size $\mu$ reaches larger values of $\mu_{b}$. This, on practical point of view, implies that relatively curved panels seldom experience bifurcation buckling response.

\subsection{Thermal postbuckling of panels under preexisting external pressure}

In the following, thermal postbuckling behavior of FG-CNTRC doubly curved panels under preexisting external pressure and subjected to uniform temperature rise will be analyzed. The effects of external pressure on thermal postbuckling paths are shown in Fig. 9 depicted with four different values of $q$. It is interesting to notice that thermally loaded panels exhibit a quasibifurcation buckling response due to the presence of preexisting external pressure. This phenomenon can be interpreted that external pressure makes the panel deflected inwards and temperature must reach a definite value for which panel surfaces return their initial state. In addition, it is worth to notice that thermal load carrying capacity of the panel is better when preexisting external pressure is higher. Fig. 10 considers the effects of total volume fraction of CNTs on thermal postbuckling response of FG-CNTRC panels under preex-

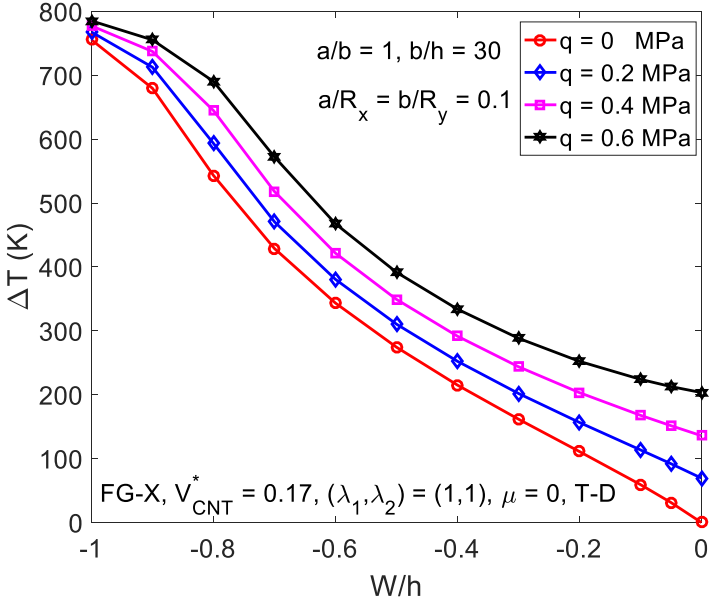

Fig. 9. Effects of preexisting external pressure on thermal postbuckling responses of FG-CNTRC doubly curved panels isting external pressure. It is evident that, contrast to situation shown in Fig. 4, postbuckling paths are lowered when $V_{C N T}^{*}$ is increased. In other words, under combined action of external pressure and

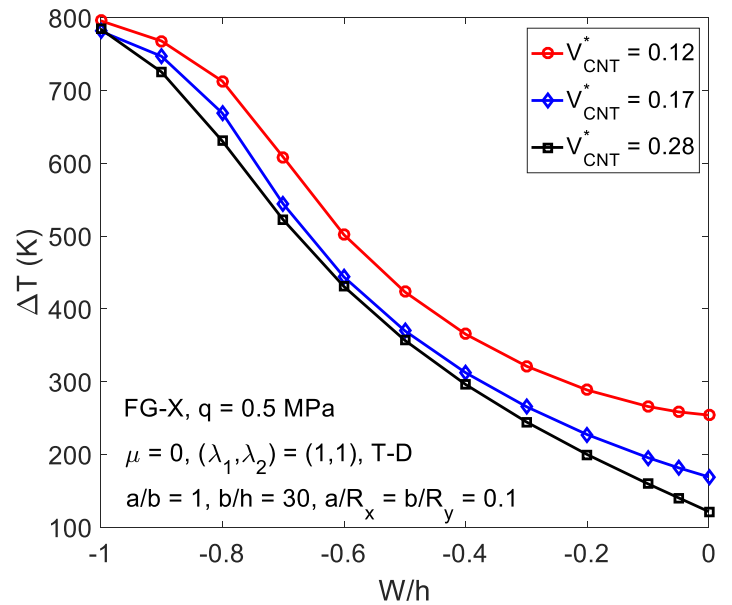

Fig. 10. Effects of $V_{C N T}^{*}$ on thermal postbuckling response of FG-CNTRC curved panels with preexisting external pressure

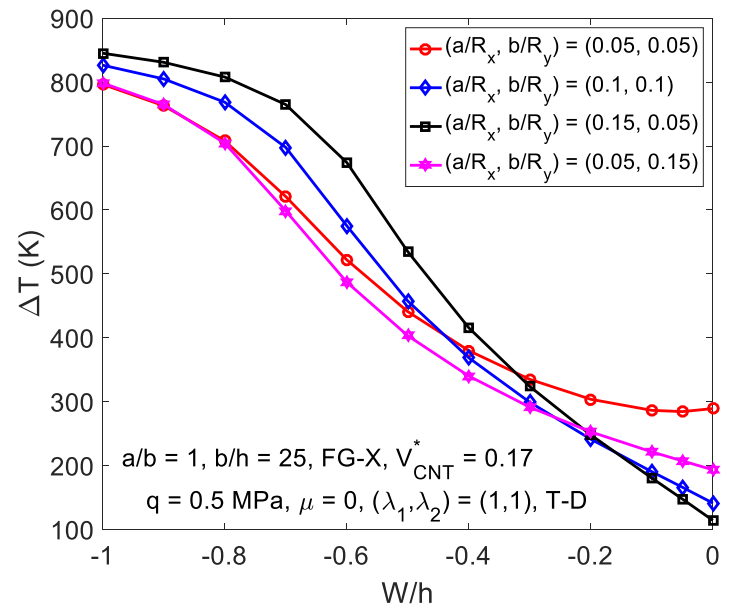

Fig. 11. Effects of curvature on thermal postbuckling response of FG-CNTRC doubly curved panels under preexisting external pressure 
thermal loads, high volume percentage of CNTs has deteriorative influences on the loading carrying capability of the panels.

Finally, the effects of curvature ratios on thermal postbuckling behavior of FG-CNTRC doubly curved panels under preexisting external pressure are assessed in Fig. 11. This figure indicates that "bifurcation-point" pressure is higher for more shallow panel. This means that more shallow panels are more deflected inwards prior to application of thermal load. However, in deep region of negative deflection, postbuckling path is higher as the panel is more curved. Fig. 11 also explores that curvature in the $x$ direction (i.e. the longitudinal direction of CNTs) has more significant influence than curvature in the $y$ direction.

\section{CONCLUDING REMARKS}

For the first time, an analytical investigation on thermal postbuckling behavior of thick FG-CNTRC doubly curved panels with and without preexisting external pressure has been presented. To reflect more practical situations, both temperature dependent properties and elasticity of tangential edge constrains are taken into consideration. The panels are modelled within the framework of a higher order shear deformation shell theory including initial geometrical imperfection. The study reveals that thermally loaded curved panels can exhibit a quasi-bifurcation buckling response due to presence of preexisting external pressure. For the most part, geometrically perfect curved panels are deflected outwards at the onset of heating. This results from curved geometry and moment-related prebuckling state. In some special situations in which imperfection size satisfies particular conditions, geometrically imperfect curved panels may experience a bifurcation type buckling response. The results also indicate that thermal postbuckling response of doubly curved panels are strongly influenced by tangential edge constraints and curvature ratios, especially constraint and curvature in longitudinal direction of CNTs. It is hoped that this study has contribution for a better understanding of thermal postbuckling behavior of composite and nanocomposite doubly curved panels.

\section{ACKNOWLEDGMENT}

This research is funded by Vietnam National Foundation for Science and Technology Development (NAFOSTED) under grant number 107.02-2019.318.

\section{REFERENCES}

[1] E. T. Thostenson, C. Li, and T. W. Chou. Nanocomposites in context. Composites Science and Technology, 65, (3-4), (2005), pp. 491-516. https://doi.org/10.1016/j.compscitech.2004.11.003.

[2] H. S. Shen. Nonlinear bending of functionally graded carbon nanotube-reinforced composite plates in thermal environments. Composite Structures, 91, (1), (2009), pp. 9-19. https://doi.org/10.1016/j.compstruct.2009.04.026.

[3] E. García-Macías, L. Rodriguez-Tembleque, R. Castro-Triguero, and A. Sáez. Buckling analysis of functionally graded carbon nanotube-reinforced curved panels under axial compression and shear. Composites Part B: Engineering, 108, (2017), pp. 243-256. https://doi.org/10.1016/j.compositesb.2016.10.002.

[4] S. Zghal, A. Frikha, and F. Dammak. Mechanical buckling analysis of functionally graded power-based and carbon nanotubes-reinforced composite plates and curved panels. Composites Part B: Engineering, 150, (2018), pp. 165-183. https://doi.org/10.1016/j.compositesb.2018.05.037.

[5] H. S. Shen. Postbuckling of nanotube-reinforced composite cylindrical panels resting on elastic foundations subjected to lateral pressure in thermal environments. Engineering Structures, 122, (2016), pp. 174-183. https://doi.org/10.1016/j.engstruct.2016.05.004.

[6] L. T. N. Trang and H. V. Tung. Buckling and postbuckling of carbon nanotube-reinforced composite cylindrical panels subjected to axial compression in thermal environments. Vietnam Journal of Mechanics, 40, (1), (2018), pp. 47-61. https://doi.org/10.15625/0866-7136/10088.

[7] H. V. Tung and L. T. N. Trang. Imperfection and tangential edge constraint sensitivities of thermomechanical nonlinear response of pressure-loaded carbon nanotube-reinforced composite cylindrical panels. Acta Mechanica, 229, (5), (2018), pp. 1949-1969. https://doi.org/10.1007/s00707-017-2093-z.

[8] L. T. N. Trang and H. V. Tung. Thermomechanical nonlinear analysis of axially compressed carbon nanotubereinforced composite cylindrical panels resting on elastic foundations with tangentially restrained edges. Journal of Thermal Stresses, 41, (4), (2018), pp. 418-438. https://doi.org/10.1080/01495739.2017.1409093. 
[9] H. S. Shen. Postbuckling of nanotube-reinforced composite cylindrical shells in thermal environments, Part I: Axially-loaded shells. Composite Structures, 93, (8), (2011), pp. $2096-2108$. https://doi.org/10.1016/j.compstruct.2011.02.011.

[10] H. S. Shen. Postbuckling of nanotube-reinforced composite cylindrical shells in thermal environments, Part II: Pressure-loaded shells. Composite Structures, 93, (10), (2011), pp. $2496-2503$. https://doi.org/10.1016/j.compstruct.2011.04.005.

[11] H. S. Shen and C. L. Zhang. Thermal buckling and postbuckling behavior of functionally graded carbon nanotube-reinforced composite plates. Materials $\mathcal{E}$ Design, 31, (7), (2010), pp. 3403-3411. https://doi.org/10.1016/j.matdes.2010.01.048.

[12] M. Mirzaei and Y. Kiani. Thermal buckling of temperature dependent FG-CNT reinforced composite plates. Meccanica, 51, (9), (2016), pp. 2185-2201. https://doi.org/10.1007/s11012-015-0348-0.

[13] Y. Kiani. Thermal buckling of temperature-dependent FG-CNT-reinforced composite skew plates. Journal of Thermal Stresses, 40, (11), (2017), pp. 1442-1460. https://doi.org/10.1080/01495739.2017.1336742.

[14] Y. Kiani. Thermal post-buckling of FG-CNT reinforced composite plates. Composite Structures, 159, (2017), pp. 299-306. https://doi.org/10.1016/j.compstruct.2016.09.084.

[15] Y. Kiani. Thermal post-buckling of temperature dependent sandwich plates with FG-CNTRC face sheets. Journal of Thermal Stresses, 41, (7), (2018), pp. 866-882. https://doi.org/10.1080/01495739.2018.1425645.

[16] H. V. Tung. Thermal buckling and postbuckling behavior of functionally graded carbon-nanotube-reinforced composite plates resting on elastic foundations with tangential-edge restraints. Journal of Thermal Stresses, 40 , (5), (2017), pp. 641-663. https://doi.org/10.1080/01495739.2016.1254577.

[17] H. V. Tung and L. T. N. Trang. Thermal postbuckling of shear deformable CNT-reinforced composite plates with tangentially restrained edges and temperature-dependent properties. Journal of Thermoplastic Composite Materials, 33, (1), (2020), pp. 97-124. https://doi.org/10.1177/0892705718804588.

[18] V. T. Long and H. V. Tung. Thermal postbuckling behavior of CNT-reinforced composite sandwich plate models resting on elastic foundations with tangentially restrained edges and temperaturedependent properties. Journal of Thermoplastic Composite Materials, 33, (10), (2020), pp. 1396-1428. https://doi.org/10.1177/0892705719828789.

[19] V. T. Long and H. V. Tung. Thermomechanical postbuckling behavior of CNT-reinforced composite sandwich plate models resting on elastic foundations with elastically restrained unloaded edges. Journal of Thermal Stresses, 42, (5), (2019), pp. 658-680. https://doi.org/10.1080/01495739.2019.1571972.

[20] H. S. Shen. Thermal buckling and postbuckling behavior of functionally graded carbon nanotubereinforced composite cylindrical shells. Composites Part B: Engineering, 43, (3), (2012), pp. 1030-1038. https://doi.org/10.1016/j.compositesb.2011.10.004.

[21] P. T. Hieu and H. V. Tung. Thermal buckling and postbuckling of CNT-reinforced composite cylindrical shell surrounded by an elastic medium with tangentially restrained edges. Journal of Thermoplastic Composite Materials, (2019). https://doi.org/10.1177/0892705719853611.

[22] M. Mirzaei and Y. Kiani. Thermal buckling of temperature dependent FG-CNT reinforced composite conical shells. Aerospace Science and Technology, 47, (2015), pp. 42-53. https://doi.org/10.1016/j.ast.2015.09.011.

[23] P. T. Hieu and H. V. Tung. Thermal and thermomechanical buckling of shear deformable FG-CNTRC cylindrical shells and toroidal shell segments with tangentially restrained edges. Archive of Applied Mechanics, (2020), pp. 1-18. https://doi.org/10.1007/s00419-020-01682-7.

[24] H. S. Shen and Y. Xiang. Postbuckling of pressure-loaded nanotube-reinforced composite doubly curved panels resting on elastic foundations in thermal environments. International Journal of Mechanical Sciences, 107, (2016), pp. 225-234. https://doi.org/10.1016/j.ijmecsci.2016.01.004.

[25] L. T. N. Trang and H. V. Tung. Thermomechanical nonlinear stability of pressure-loaded CNT-reinforced composite doubly curved panels resting on elastic foundations. Nonlinear Engineering, 8, (1), (2019), pp. 582-596. https://doi.org/10.1515/nleng-2018-0077.

[26] L. T. N. Trang and H. V. Tung. Thermomechanical nonlinear stability of pressure-loaded functionally graded carbon nanotube-reinforced composite doubly curved panels with tangentially restrained edges. Proceedings of the Institution of Mechanical Engineers, Part C: Journal of Mechanical Engineering Science, 233, (16), (2019), pp. 58485859. https://doi.org/10.1177/0954406219856374.

[27] H. V. Tung and N. D. Duc. Nonlinear response of shear deformable FGM curved panels resting on elastic foundations and subjected to mechanical and thermal loading conditions. Applied Mathematical Modelling, 38, (11-12), (2014), pp. 2848-2866. https://doi.org/10.1016/j.apm.2013.11.015.

[28] H. V. Tung. Postbuckling of thick FGM cylindrical panels with tangential edge constraints and temperature dependent properties. Vietnam Journal of Mechanics, 38, (2), (2016), pp. 123-140. https://doi.org/10.15625/0866$7136 / 38 / 2 / 7066$.

[29] H. S. Shen and H. Wang. Thermal postbuckling of FGM cylindrical panels resting on elastic foundations. Aerospace Science and Technology, 38, (2014), pp. 9-19. https://doi.org/10.1016/j.ast.2014.07.009. 
[30] K. Mehar, S. K. Panda, Y. Devarajan, and G. Choubey. Numerical buckling analysis of graded CNT-reinforced composite sandwich shell structure under thermal loading. Composite Structures, 216, (2019), pp. 406-414. https://doi.org/10.1016/j.compstruct.2019.03.002.

[31] H. S. Shen and Y. Xiang. Thermal postbuckling of nanotube-reinforced composite cylindrical panels resting on elastic foundations. Composite Structures, 123, (2015), pp. 383-392. https://doi.org/10.1016/j.compstruct.2014.12.059.

[32] L. T. N. Trang and H. V. Tung. Thermally induced postbuckling of higher order shear deformable CNT-reinforced composite flat and cylindrical panels resting on elastic foundations with elastically restrained edges. Mechanics Based Design of Structures and Machines, (2020), pp. 1-24. https://doi.org/10.1080/15397734.2020.1785312.

[33] J. N. Reddy and C. F. Liu. A higher-order shear deformation theory of laminated elastic shells. International Journal of Engineering Science, 23, (3), (1985), pp. 319-330. https://doi.org/10.1016/0020-7225(85)90051-5.

\section{APPENDIX A}

The details of the coefficients $a_{k 3}(k=1 \div 4)$ in the Eq. (22) are the following

$$
\begin{aligned}
a_{13}= & \frac{\pi^{3}}{B_{h}^{3}}\left\{\bar{a}_{11} \bar{B}_{1}^{*} m^{3} B_{a}^{3}+\bar{a}_{21} \bar{B}_{1}^{*} m n^{2} B_{a}+\bar{a}_{31} \bar{B}_{2}^{*} m^{2} n B_{a}^{2}+\bar{a}_{41} \bar{B}_{2}^{*} n^{3}\right. \\
& +\frac{\bar{a}_{51} m^{2} n^{2} \pi B_{a}^{2}}{\bar{b}_{14} B_{h}}\left[\left(n^{2} R_{a x}+m^{2} B_{a} R_{b y}\right) \frac{\pi^{2} B_{a}}{B_{h}^{3}} \bar{b}_{24}-\bar{b}_{34}\right] \\
& +\frac{\pi}{B_{h}}\left(\bar{a}_{61} m^{4} B_{a}^{4}+\bar{a}_{71} m^{2} n^{2} B_{a}^{2}+\bar{a}_{81} n^{4}\right) \\
& \left.-\frac{B_{a}}{\pi \bar{b}_{14}}\left[\left(n^{2} R_{a x}+m^{2} B_{a} R_{b y}\right) \frac{\pi^{2} B_{a}}{B_{h}^{3}} \bar{b}_{24}-\bar{b}_{34}\right]\left(n^{2} R_{a x}+m^{2} B_{a} R_{b y}\right)\right\}, \\
a_{23}= & 32 \frac{m n \pi^{2} B_{a}^{2}}{3 \bar{b}_{14} B_{h}^{4}} \gamma_{m} \gamma_{n}\left[\left(n^{2} R_{a x}+m^{2} B_{a} R_{b y}\right) \frac{\pi^{2} B_{a}}{B_{h}^{3}} \bar{b}_{24}-\bar{b}_{34}\right], \\
a_{33}= & \frac{2 \gamma_{m} \gamma_{n}}{3 m n B_{h}^{3}}\left(\frac{m^{2} B_{a}^{3}}{\bar{a}_{32}} R_{a x}+\frac{n^{2}}{\bar{a}_{12}} R_{b y}\right), \\
a_{43}= & -\left(\frac{n^{2}}{\bar{a}_{12} m^{2}}+\frac{m^{2} B_{a}^{4}}{\bar{a}_{32} n^{2}}\right) \frac{m^{2} n^{2} \pi^{4}}{16 B_{h}^{4}} .
\end{aligned}
$$

in which

$$
\begin{aligned}
& \left(\bar{a}_{11}, \bar{a}_{21}, \bar{a}_{31}, \bar{a}_{41}, \bar{a}_{61}, \bar{a}_{71}, \bar{a}_{81}\right)=\frac{1}{h^{3}}\left(a_{11}, a_{21}, a_{31}, a_{41}, a_{61}, a_{71}, a_{81}\right), \\
& \bar{a}_{51}=\frac{a_{51}}{h} \\
& \left(\bar{a}_{12}, \bar{a}_{32}\right)=h\left(a_{12}, a_{32}\right), \\
& \bar{b}_{14}=b_{14} h^{3}, \quad \bar{b}_{34}=b_{34} h \\
& \bar{B}_{1}^{*}=B_{1}^{*} h, \quad \bar{B}_{2}^{*}=B_{2}^{*} h .
\end{aligned}
$$

\section{APPENDIX B}

The detailed definitions of coefficients $a_{i 6}$ and $a_{i 7}(i=1 \div 3)$ in the Eqs. (24) are

$$
\begin{aligned}
& a_{16}=\frac{\bar{c}_{1}\left(1-\bar{c}_{2} a_{25}\right) a_{34}+\bar{c}_{1} \bar{c}_{2} a_{24} a_{35}}{\left(1-\bar{c}_{1} a_{14}\right)\left(1-\bar{c}_{2} a_{25}\right)-\bar{c}_{1} \bar{c}_{2} a_{15} a_{24}}, \quad a_{26}=\frac{\bar{c}_{1}\left(1-\bar{c}_{2} a_{25}\right) a_{44}+\bar{c}_{1} \bar{c}_{2} a_{24} a_{45}}{\left(1-\bar{c}_{1} a_{14}\right)\left(1-\bar{c}_{2} a_{25}\right)-\bar{c}_{1} \bar{c}_{2} a_{15} a_{24}}, \\
& a_{36}=\frac{\bar{c}_{1}\left(1-\bar{c}_{2} a_{25}\right) a_{54}+\bar{c}_{1} \bar{c}_{2} a_{24} a_{55}}{\left(1-\bar{c}_{1} a_{14}\right)\left(1-\bar{c}_{2} a_{25}\right)-\bar{c}_{1} \bar{c}_{2} a_{15} a_{24}}, \quad a_{17}=\frac{\left(1-\bar{c}_{1} a_{14}\right) \bar{c}_{2} a_{35}+\bar{c}_{1} \bar{c}_{2} a_{15} a_{34}}{\left(1-\bar{c}_{1} a_{14}\right)\left(1-\bar{c}_{2} a_{25}\right)-\bar{c}_{1} \bar{c}_{2} a_{15} a_{24}}, \\
& a_{27}=\frac{\left(1-\bar{c}_{1} a_{14}\right) \bar{c}_{2} a_{45}+\bar{c}_{1} \bar{c}_{2} a_{15} a_{44}}{\left(1-\bar{c}_{1} a_{14}\right)\left(1-\bar{c}_{2} a_{25}\right)-\bar{c}_{1} \bar{c}_{2} a_{15} a_{24}}, \quad a_{37}=\frac{\left(1-\bar{c}_{1} a_{14}\right) \bar{c}_{2} a_{55}+\bar{c}_{1} \bar{c}_{2} a_{15} a_{54}}{\left(1-\bar{c}_{1} a_{14}\right)\left(1-\bar{c}_{2} a_{25}\right)-\bar{c}_{1} \bar{c}_{2} a_{15} a_{24}},
\end{aligned}
$$


in which

$$
\begin{aligned}
\left(\bar{c}_{1}, \bar{c}_{2}\right)= & \frac{1}{h}\left(c_{1}, c_{2}\right), \quad a_{14}=-\frac{1}{\left(1-v_{12} v_{21}\right) \bar{e}_{11}}, a_{24}=\frac{v_{21}}{\left(1-v_{12} v_{21}\right) \bar{e}_{21}}, \\
a_{34}= & -\frac{4 \gamma_{m} \gamma_{n}}{m n \pi^{2}\left(1-v_{12} v_{21}\right) \bar{e}_{11} \bar{e}_{21}}\left\{\frac{v_{21} \bar{e}_{11} m^{2} \pi^{2} B_{a}^{2}-\bar{e}_{21} n^{2} \pi^{2}}{B_{h}^{2} \bar{b}_{14}}\left[\left(n^{2} R_{a x}+m^{2} B_{a} R_{b y}\right) \frac{\pi^{2} B_{a} \bar{b}_{24}}{B_{h}^{3}}-\bar{b}_{34}\right]\right. \\
& -\left(v_{12} v_{21} \bar{e}_{11} \bar{e}_{22}-\bar{e}_{12} \bar{e}_{21}\right) m \pi \bar{B}_{1}^{*} \frac{B_{a}}{B_{h}}-\left(\bar{e}_{11} \bar{e}_{22}-\bar{e}_{12} \bar{e}_{21}\right) n \pi \bar{B}_{2}^{*} \frac{v_{21}}{B_{h}} \\
& \left.+\frac{4 m \pi B_{a}}{3 B_{h}}\left(\bar{B}_{1}^{*}+m \pi \frac{B_{a}}{B_{h}}\right)\left(v_{12} v_{21} \bar{e}_{11} \bar{e}_{24}-\bar{e}_{14} \bar{e}_{21}\right)+\frac{4 v_{21} n \pi}{3 B_{h}}\left(\bar{B}_{2}^{*}+\frac{n \pi}{B_{h}}\right)\left(\bar{e}_{11} \bar{e}_{24}-\bar{e}_{14} \bar{e}_{21}\right)\right\} \\
& -\frac{4 \gamma_{m} \gamma_{n} B_{a} R_{a x}}{m n \pi^{2} B_{h}}, \\
a_{44}= & m^{2} \pi^{2} \frac{B_{a}^{2}}{8 B_{h}^{2}}, a_{54}=-\frac{\left(\bar{e}_{21} \bar{e}_{11 T}-v_{21} \bar{e}_{11} \bar{e}_{21 T}\right)}{\left(1-v_{12} v_{21}\right) \bar{e}_{11} \bar{e}_{21}}, a_{15}=\frac{v_{12}}{\left(1-v_{12} v_{21}\right) \bar{e}_{11}}, a_{25}=-\frac{1}{\left(1-v_{12} v_{21}\right) \bar{e}_{21}}, \\
a_{35}= & -\frac{4 \gamma_{m} \gamma_{n}}{m n \pi^{2}\left(1-v_{12} v_{21}\right) \bar{e}_{11} \bar{e}_{21}}\left\{\frac{\left(\bar{e}_{21} v_{12} n^{2} \pi^{2}-\bar{e}_{11} m^{2} \pi^{2} B_{a}^{2}\right)}{B_{h}^{2} \bar{b}_{14}}\left[\left(n^{2} R_{a x}+m^{2} B_{a} R_{b y}\right) \frac{\pi^{2} B_{a}}{B_{h}^{3}} \bar{b}_{24}-\bar{b}_{34}\right]\right. \\
& -\left(\bar{e}_{12} \bar{e}_{21}-\bar{e}_{11} \bar{e}_{22}\right) \bar{B}_{1}^{*} v_{12} m \pi \frac{B_{a}}{B_{h}}-\left(v_{12} v_{21} \bar{e}_{12} \bar{e}_{21}-\bar{e}_{11} \bar{e}_{22}\right) \bar{B}_{2}^{*} \frac{n \pi}{B_{h}} \\
& \left.+v_{12} m \pi \frac{4 B_{a}}{3 B_{h}}\left(\bar{B}_{1}^{*}+m \pi \frac{B_{a}}{B_{h}}\right)\left(\bar{e}_{14} \bar{e}_{21}-\bar{e}_{11} \bar{e}_{24}\right)+\frac{4 n \pi}{3 B_{h}}\left(\bar{B}_{2}^{*}+\frac{n \pi}{B_{h}}\right)\left(v_{12} v_{21} \bar{e}_{14} \bar{e}_{21}-\bar{e}_{24} \bar{e}_{11}\right)\right\} \\
& -\frac{4 \gamma_{m} \gamma_{n} R_{b y}}{m n \pi^{2} B_{h}}, \\
a_{45}= & \frac{n^{2} \pi^{2}}{8 B_{h}^{2}}, \quad a_{55}=-\frac{\left(\bar{e}_{11} \bar{e}_{21 T}-v_{12} e_{21} \bar{e}_{11 T}\right)}{\left(1-v_{12} v_{21}\right) \bar{e}_{11} \bar{e}_{21}} .
\end{aligned}
$$

and

$$
\begin{aligned}
& \left(\bar{e}_{11}, \bar{e}_{21}, \bar{e}_{11 T}, \bar{e}_{21 T}\right)=\frac{1}{h}\left(e_{11}, e_{21}, e_{11 T}, e_{21 T}\right), \\
& \left(\bar{e}_{12}, \bar{e}_{22}\right)=\frac{1}{h^{2}}\left(e_{12}, e_{22}\right), \\
& \left(\bar{e}_{14}, \bar{e}_{24}\right)=\frac{1}{h^{4}}\left(e_{14}, e_{24}\right) .
\end{aligned}
$$

